\title{
Tuning Co valence state in cobalt oxyhydrate superconductor by post reduction
}

\author{
Zhi Ren, Jian-lin Luo , Zhu-an Xu, Guang-han Cao*
}

Department of Physics, Zhejiang University, Hangzhou 310027, People's Republic of China

"Beijing National Laboratory of Condensed Matter Physics, Institute of Physics, Chinese Academy of Sciences, Beijing 10080, People's Republic of China 


\section{SUPPORTING INFORMATION PARAGRAPH}

The thermal analysis for samples treated in $0.1 \mathrm{M}$ and $1 \mathrm{M} \mathrm{NaOH}$ is shown in figure $\mathrm{S} 1$. According to our previous analysis, ${ }^{1}$ a small weight-loss around $481 \mathrm{~K}$ in figure $\mathrm{S}$ 1(a) accompanied by an endothermal peak is due to the loss of water from the incorporated hydronium ions. Therefore, the hydronium content is then determined to be $0.04(1)$ by the TG data. In figure $S$ 1(b) for the sample treated in $1 \mathrm{M} \mathrm{NaOH}$, in contrast, such a weight loss cannot be detected, which indicates that the hydronium content for the sample is, even if exists, negligible.

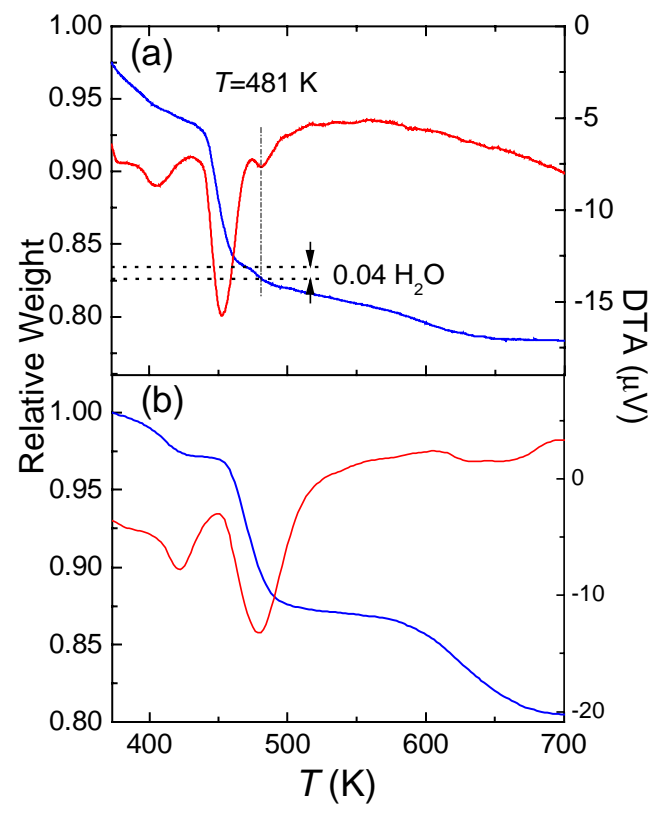

Figure S 1 . Thermal analysis of the cobalt oxyhydrate superconductor post-reduced in (a) $0.1 \mathrm{M} \mathrm{NaOH}$ (b) $1 \mathrm{M} \mathrm{NaOH}$.

(1) Ren, Z.; Wang, Y. W.; Liu, S.; Wang, J.; Xu, Z. A.; Cao, G. H. Chem. Mater. 2005, 17, 1501. 